\title{
Special issue on energy-efficient optical networks
}

\author{
Paolo Monti $^{1}$ - Annalisa Morea ${ }^{2}$ - Ampalavanapillai Nirmalathas ${ }^{3}$. \\ Vinod M. Vokkarane ${ }^{4}$
}

Published online: 15 July 2015

(C) Springer Science+Business Media New York 2015

Communication networks continue to experience an increase in traffic volumes, number of connected devices, and users. For this reason, the energy consumption due to the communication infrastructure became an important parameter to be carefully considered and optimized during both network design and live operations. In this paradigm, optical transmission technologies offer the possibility to significantly lower the overall energy consumption level of communication networks. For this reason, there is a growing interest on how to take advantage of the energy-saving opportunities provided by optical networks. In addition, there are also parallel research efforts targeting the improvement of the energy efficiency of optical networks themselves.

Despite this very vibrant research area, there are still a number of open questions that need to be addressed and new research directions have been recently opened in the field of energy-efficient optical networks. For example, the energy consumption levels in an optical network cannot be lowered at the expense of other network performance parameters (e.g., connection survivability, latency, average component reliability levels). As a result, energy-efficient schemes in optical networks have now been tailored to limit their impact on the Quality of Service (QoS) level of the provisioned connections. New network architectures have also been considered

Paolo Monti

pmonti@kth.se

1 School of Information and Communication Technology, KTH Royal Institute of Technology, 16440 Kista, Sweden

2 Alcatel-Lucent Bell Labs, Nozay, France

3 Melbourne Networked Society Institute, The University of Melbourne, Melbourne, VIC 3010, Australia

4 Department of Electrical and Computer Engineering, University of Massachusetts, Lowell, MA 01854, USA both in the core (e.g., optical cloud) and in the access (e.g., fixed mobile convergence) to enable a new generation of network services where end-to-end energy optimization is crucial. All these efforts are the proof of a still flourishing research area looking for cutting-edge solutions toward energy efficiency in optical networks.

This special issue solicited submissions of original works and survey papers on all topics related to recent advances in the field of energy-efficient optical networks. The special issue consists of eight papers. The first three are invited papers from renowned experts in the field of green networking. The other five are regular contributed papers. All papers included in this special issue underwent the same thorough review process. A brief summary of the topic addressed by each paper is provided next.

The paper "Analysis of the Energy Consumption in Telecom Operator Networks" by C. Lange discusses and explains the energy consumption of a countrywide mid-European incumbent operator network with fixed and mobile radiotelephony in addition to broadband services. To operate a large-scale telecommunication network, electricity is not only needed for powering its network elements. Everyday operations like installation, maintenance, and management activities also need energy in different forms. Examples are heating, air-conditioning, and lighting of workspaces/offices as well as truck rolls for installation, maintenance and repair, in addition to customer and service-related administration and support activities. The analysis presented in the paper shows that the legacy and access network parts, with predominantly electrical transmission and processing, require large amounts of electricity, whereas the aggregation, core, and optical transport networks require only comparably smaller electricity shares.

The paper "Energy Consumption Modeling of Optical Networks" by K. Hinton et al. provides a condensed overview 
of a general "first-order approximation" bottom-up power consumption model of telecommunication networks and services. A motivation for this work is to provide an intuitive introduction to telecommunication networks and service energy consumption modeling without having to rely onto detailed network equipment information, which is typically very difficult to acquire. For this reason, the model provided in the paper is based on typical equipment data (available from vendor equipment data) and simple network architecture parameters (such as number of hops). The model presented in the paper is tested to construct power consumption estimates for a wide range of network scenarios including: (i) customer premise equipment, (ii) access, edge, and core networks, and (iii) services provided over a network.

The paper "How Sleep Modes and Traffic Demands Affect the Energy Efficiency in Optical Access Networks" by B. Lannoo et al. presents a detailed evaluation of the energy consumption of different next- generation optical access (NGOA) technologies. As the access part consumes a major fraction of the energy consumption in today's fiber-to-thehome (FTTH)-based telecommunication networks, the paper analyzes the effects of (i) introducing low-power modes (e.g., sleep and doze modes) in the various NGOA technologies and (ii) the effect of using optimal split ratios adjusted to the traffic demands so that the energy consumption is optimized for the desired Quality of Service (QoS) level.

The paper "QoS-Aware Energy-efficient Mechanism for Sleeping Mode ONUs in Enhanced EPON" by A. Nikoukar et al. introduces an enhanced Ethernet Passive Optical Network (EPON) architecture and a QoS-aware energy-saving mechanism able to reduce the energy consumption of optical network units (ONUs) while guaranteeing an overall QoS metric based on the ITU-T standard requirements. To achieve energy savings in the upstream/downstream direction, two sleep durations are defined for the ONU's transmitter and receiver resulting in four ONU states: active, transmission, doze, and sleep. The proposed scheme improves the energy efficiency by $44 \%$, on average, while fulfilling the QoS metrics in terms of packet loss, delay, jitter, and buffer requirements.

The paper "Adaptive State Transition Control for Energyefficient Gigabit-Capable Passive Optical Networks" by S. $\mathrm{S}$. W. Lee et al. investigates the power management problems that affect Gigabit-capable Passive Optical Networks (GPONs). The paper shows that due to the presence of surge currents, the frequency of transitions from a power saving state to a full power state has an impact on the overall network power consumption. Based on these considerations, the paper proposes an adaptive control scheme for doze mode in GPON systems. The proposed approach uses an optimal load threshold to determine when to turn an optical network unit (ONU) and a neural network-based scheme to determine, on the other hand, when to turn an ONU in a power saving state.
Simulation results indicate that the proposed adaptive control scheme can achieve power consumption levels that are very close to the theoretical optimum.

The paper "Energy Efficiency Analysis of Aggregation Mechanisms in IEEE 802.11n Radio-over-Fiber-based Distributed Antenna Systems" by S. Deronne et al. focuses on the energy efficiency of 802.11n Radio-over-Fiber (RoF) Distributed Antenna Systems (DAS) architectures and provides a methodology based on ns-3 to evaluate and optimize the energy consumption in those environments. The results confirm that there exist an optimal number of distributed antennas (in terms of energy efficiency) for a given scenario. Furthermore, the paper shows that aggregation mechanisms included in IEEE 802.11n enable further improvements of the energy efficiency of RoF-based DAS architectures.

The paper "Facing the Traffic Explosion in Metro Transport Networks with Energy-sustainable Architectures" by E. Bonetto et al. addresses a scenario where the implementation of new content distribution solutions will speed up the traffic increase in metro networks. In such a paradigm, the paper proposes a set of metro network design solutions based on Integer Linear Programming formulations where different architectures for a metro network are considered. The aim of the paper is to understand which architecture can better face the considered traffic evolution. The comparison is performed considering the energy consumption, the network setup and management, and the Quality of Service performance ensured by each architecture.

The paper "Energy-efficiency vs. resilience" by P. Cholda et al. proposes to solve the trade-off between energy efficiency and resilience with a focus on business mechanisms, where risk engineering is used as a foundation. The proposed approach is used in networks with energy profiles supporting sleep mode. An effective heuristic is used to provision traffic flows. The paper shows that the energy efficiency performance in a network is substantially independent of the recovery methods selected for risk mitigation. The paper also demonstrates that backup resources can be switched off while not in use without having a considerable impact from a financial viewpoint.

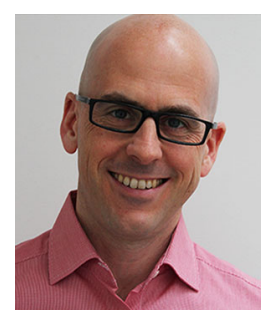

Paolo Monti received a Ph.D. in Electrical Engineering (2005) from The University of Texas at Dallas (UTD). From 2006 to 2008, he worked as a Research Associate of the Open Networking Advance Research $(\mathrm{OpN}$ eAR) Lab at UTD. He joined KTH Royal Institute of Technology in September 2008 where he is currently an Assistant Professor, and the deputy director of the Optical Networks Laboratory (ONLab). Dr. Monti published more than 100 papers in peer-reviewed international journals and conferences. He is serving on the editorial boards of the IEEE Journal on Selected Areas in Communications-Series on Green Communications and Networking and of the Springer Photonic Network Communications journal. Dr. 
Monti was one of the Guest Editors of the Special Issue on "Green Networking and Computing" of the Journal of High Speed Networks. He regularly participates in the TPCs of all major IEEE conferences including IEEE Globecom and ICC where he also co-chaired a number of workshops on WDM network survivability (ICC 2012) and green broadband access (ICC 2013, Globecom 2014, ICC 2015). Dr. Monti was the TPC co-chair of ONDM 2014 and served/serves as TPC coChair for the Symposium on Optical and Grid Computing in ICNC 2014 and ICNC 2016. His main research interests are within the networking aspects of all-optical networks (including their energy performance), while part of his work recently focused on the architectural and control challenges posed by next-generation mobile communication (i.e., 5G) on optical transport networks. Dr. Monti is a Senior Member of the IEEE.

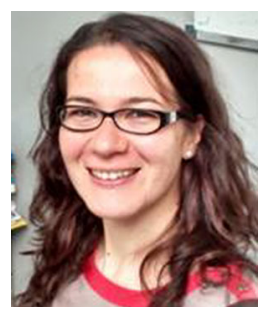

Annalisa Morea graduated in telecommunication from Politecnico di Torino, Italy, and from Ecole Nationale Supérieure des Télécommunications (ENST), France. She then received a Ph.D. degree in computer science and networks from ENST Paris, carrying out her work at the laboratories of France Telecom R\&D in Lannion, France. She joined Alcatel-Lucent in 2006. From 2006 to 2014, she worked in Alcatel-Lucent Bell Labs, France, as a research engineer in the Optical Networks Department, and in 2015, she joined Alcatel-Lucent Italy as software engineer. Her work mostly focuses on the dimensioning of transparent optical networks in order to estimate the interest of new technologies, such as elastic interfaces, and new network solutions, like multilayer networking, for mid- and longterm requirements. By these studies, she evaluates the performance of the proposed networks in terms of capital and operational expenditures (CAPEX/OPEX), and environmental impact. She authored and co-authored more than 50 peer-reviewed articles published in major journals and conferences into the optical communication domain, and filed more than 20 patents. She participated to various national and European collaborative projects (among which ANRT RYTHME dedicated to impairment-aware transparent optical networks and performance estimation issues, Celtic EO-NET, FP7 IDEALIST and CELTIC + SASER dedicated to elastic optical networks); she is a member of diverse technical committees: particularly in 2015 , she was chair of the subcommittee N3 ("Network architectures, techno-economics, and design tradeoffs") at OFC (one of the main international optical telecommunication conferences), and reviewer of IEEE and OSA journal articles. Since 2015 , she is part of the Associate Editors of Journal of Optical Communications and Networking (JOCN).

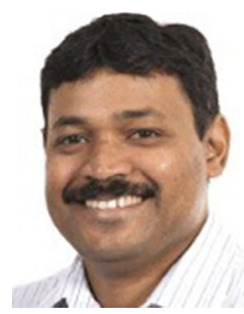

Ampalavanapillai Nirmalathas obtained his B.E. and Ph.D. in Electrical and Electronic Engineering from the University of Melbourne in 1993 and 1998, respectively. Between 2000 and 2004, he was the Director of Photonics Research Laboratory (Melbourne Node of Australian Photonics CRC) and also the Program Leader of Telecommunications Technologies Program. From 2004

to 2006, he was the Program Leader for the Network Technologies Research Program in NICTA. He was also the acting Lab Direc- tor of VRL in 2007. Between 2006 and 2008, He was the Research Group Manager of the Networked Systems Group of Victoria Research Laboratory (VRL) at the National ICT Australia (NICTA), a premier Australian research center of excellence in ICT. Since 2007, he has been a Professor of Electrical and Electronic Engineering at The University of Melbourne. Between 2010 and 2013, he was the Head of Department of Electrical and Electronic Engineering, The University of Melbourne. Between 2013 and 2014, He was the associate Director for the Institute for Broadband-Enabled Society. In 2012, he initiated and co-founded and now provides academic leadership to the Australia's first university-based start-up accelerator-Melbourne Accelerator Program, aimed at promoting entrepreneurship culture on campus. He is currently the Director of Melbourne Networked Society Institute-an interdisciplinary research institute focusing on challenges and opportunities arising from the society's transition toward a networked society. He has written more than 400 technical articles and currently holds three active international patents. His current research interests include energy-efficient telecommunications, broadband access networks, optical wireless network integration, and optical wireless interconnects. He has held many editorial roles with the IEICE Transactions in Communications, IEEE/OSA Journal of Lightwave Technology and Photonics and Networks SPIE Journal. He is a Senior Member of IEEE, a member of Optical Society of America, and a Fellow of the Institution of Engineers Australia.

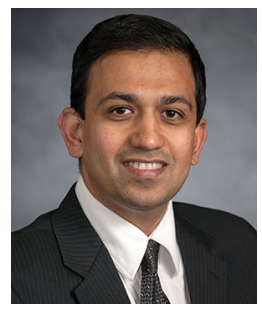

Vinod M. Vokkarane is an Associate Professor in the department of Electrical and Computer Engineering at the University of Massachusetts Lowell. Prior to this, he was an Associate Professor of Computer and Information Science at the University of Massachusetts Dartmouth from 2004. He was also a Visiting Scientist at the Claude E. Shannon Communication and Network Group, Research Laboratory of Electronics (RLE) at Massachusetts Institute of Technology (MIT) from 2011 to 2014. He received the B.E. degree with Honors in Computer Science and Engineering from the University of Mysore, India, in 1999, and the M.S. and the Ph.D. degree in Computer Science from the University of Texas at Dallas in 2001 and 2004, respectively. His primary areas of research include design and analysis of architectures and protocols for ultra-high speed networks, grid and cloud networks, and green networking. He has published more than 120 peer-reviewed journal and conference papers. He is the recipient of the UMass Dartmouth Scholar of the Year Award 2011, the UMass Dartmouth Chancellor's Innovation in Teaching Award 2010-2011, the University of Texas at Dallas Computer Science Dissertation of the Year Award 2003-2004, and the Texas Telecommunication Engineering Consortium Fellowship 2002-2003. Dr. Vokkarane is the co-author of a book, "Optical Burst Switched Networks," Springer, 2005. He is currently on the Editorial Board of IEEE/OSA Journal of Optical Communications and Networking (JOCN) and Springer Photonic Network Communications Journal and has also served as an Editor of IEEE Communications Letters and Elsevier Journal of Optical Switching and Networking. He has co-authored several Best Paper Awards, including the IEEE GLOBECOM 2005 and IEEE ANTS 2010. He has served as the Technical Program Committee Chair for the Optical Networks and Systems 